\title{
Distribuição de peixes na microbacia do Ribeirão Grande, Serra da Mantiqueira Oriental, São Paulo, Brasil
}

\author{
Francisco Manoel de Souza Braga \& Pedro de Moraes Andrade
}

Grupo de Biologia e Ecologia de Peixes (CNPq). Departamento de Zoologia, UNESP, Caixa Postal 199, 13506-900 Rio Claro, SP. (fmsbraga@rc.unesp.br)

\begin{abstract}
Fish distribution in the Ribeirão Grande Basin, Eastern Serra da Mantiqueira, São Paulo, Brazil. Eight sites were surveyed in the Ribeirão Grande system ( $\left.22^{\circ} 47^{\prime} 08^{\prime \prime} S, 45^{\circ} 28^{\prime} 17^{\prime \prime W}\right)$ from July 2001 to April 2002. Fishes were sampled four times each site, during the winter (July 2001), spring (October 2001), summer (February 2002) and autumn (April 2002). Conductivity, pH, water temperature and dissolved oxygen were measured for each site, and elevations in meters were taken by a GPS receiver. The spatial dimension was the main source of variability in the fish distribution in the Ribeirão Grande system. A total of 37 species were caught and the ten most abundant species correspond almost $90 \%$ of the total catch. Pareiorhina rudolphi was caught principally in the slope; Characidium lauroi, Trichomycterus itatiayae, Neoplecostomus microps, Astyanax scabripinnis, Taunayia bifasciata and Harttia carvalhoi were caught in the piedmont; Characidium alipioi, Imparfinis minutus and Loricariichthys sp. were caught in the plain of the valley. The sampling stations P6 and P7, located in the transition between tributaries and main channel, were considered an ecotone, where the species diversity was higher.
\end{abstract}

KEYWORDS. Fish, distribution, streams, diversity, Serra da Mantiqueira.

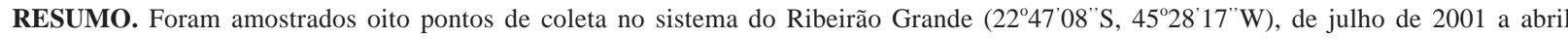
de 2002. Os peixes foram amostrados quatro vezes em cada ponto, no inverno (julho de 2001), na primavera (outubro de 2001), no verão (fevereiro de 2002) e no outono (abril de 2002). Em cada ponto obteve-se a condutividade, pH, temperatura da água e oxigênio dissolvido, a elevação foi obtida em metros utilizando-se de um receptor GPS. A dimensão da distribuição espacial foi a principal fonte de variabilidade na distribuição de peixes no sistema do Ribeirão Grande. Foi capturado um total de 37 espécies e as dez espécies mais abundantes correspondem a quase $90 \%$ do total capturado. Pareiorhina rudolphi foi capturada principalmente na encosta; Characidium lauroi, Trichomycterus itatiayae, Neoplecostomus microps, Astyanax scabripinnis, Taunaya bifasciata e Harttia carvalhoi foram capturadas no pediplano; Characidium alipioi, Imparfinis minutus e Loricariichthys sp. foram capturadas na planície do vale. Os pontos amostrais P6 e P7, localizados na transição entre tributários e o canal principal, foram considerados um ecótono, onde a diversidade de espécies foi mais alta.

PALAVRAS-CHAVE. Peixe, distribuição, riachos, diversidade, Serra da Mantiqueira.

A grande diversidade de peixes de água doce neotropicais deve-se aos processos geomorfológicos que se iniciaram no fim do Cretáceo e durante o Cenozóico, quando bacias hidrográficas foram formadas e modificadas (LUNDBERG et al., 1998). Das quatro grandes bacias fluviais reconhecidas no Brasil (Amazônica, Paraná, São Francisco e do Leste), a bacia do Leste é formada por numerosos rios que desaguam no oceano Atlântico (Menezes, 1972). Um desses rios é o Paraíba do Sul, que drena a região do Vale do Paraíba indo desaguar no mar, ao norte do Estado do Rio de Janeiro.

A Mata Atlântica é tida como uma das áreas de maior biodiversidade no mundo e ocupa o segundo lugar entre as florestas tropicais mais ameaçadas (Myers et al., 2000). Considerando as características de alta biodiversidade, altas taxas de endemismo e alta pressão antrópica, fez com que a Unesco em 1991 declarasse os remanescentes deste bioma como Patrimônio Natural da Humanidade, integrando a Serra da Mantiqueira à categoria das Reservas da Biosfera da Terra.

Segundo Menezes (1994), a fauna de peixes de riachos e de outros corpos de água da Mata Atlântica está em contato direto com a floresta, que lhes propicia proteção e alimento e pelo caráter geomorfológico, essas bacias apresentam um forte grau de endemismo de suas espécies por estarem isoladas de outras bacias.
As bacias hidrográficas que compõem a bacia do Leste caracterizam-se por terem pequenas dimensões e baixa complexidade de seu sistema fluvial, sendo que os levantamentos faunísticos são poucos e não abrangem toda bacia (BIZERRIL, 1999). Embora a ictiofauna do rio Paraíba do Sul tenha sido estudada nos últimos anos (ARAúJo, 1996; BIZERRIL, 1999), ainda são necessários estudos sobre microbacias tributárias deste rio, em especial as que descem as encostas da Serra da Mantiqueira.

Este trabalho tem como objetivos caracterizar primariamente a distribuição de peixes na microbacia do Ribeirão Grande, bem como situá-la no espaço biogeográfico da Serra da Mantiqueira.

\section{MATERIAL E MÉTODOS}

A Serra da Mantiqueira estende-se pelo leste do Estado de São Paulo, sul de Minas Gerais e sudoeste do Rio de Janeiro, sendo um divisor de águas entre as bacias do rio Grande, em Minas Gerais, e a do rio Paraíba do Sul, em São Paulo e Rio de Janeiro. Segundo Ponçano et al. (1981), a Serra da Mantiqueira é formada por escarpas elevadas e morros, nos quais o planalto mineiro termina diante do Vale do Paraíba, e encontra-se dividida nesta zona em duas porções: Serra da Mantiqueira Oriental e 
Serra da Mantiqueira Ocidental. A Serra da Mantiqueira Oriental é formada por escarpas festonadas, originárias de relevos mais íngremes entre o planalto de Campos do Jordão (SP) e sua continuação até o maciço de Itatiaia (RJ), terminando a oeste, na altura da localidade de Monteiro Lobato (SP). A partir daí, a configuração geológica da Serra da Mantiqueira muda, passando a ser considerada Serra da Mantiqueira Ocidental.

A vertente da Serra da Mantiqueira Oriental voltada para o Vale do Paraíba está constituída por numerosos fragmentos de Mata Atlântica remanescentes de intensa atividade antrópica, iniciada no século XVIII pela colonização da região e intensificada no século seguinte pelo plantio de café (DeAn, 1996). Nesta vertente destacam-se os seguintes tipos de vegetação (HUECK, 1972): campos de altitude, acima de 1800 metros; mata de neblina ou mata pluvial superior, de 1800 a 1400 metros; mata pluvial inferior, de 1400 a 600 metros e a mata semiseca do Vale do Paraíba, que há muito não existe mais, salvo alguns fragmentos em áreas fortemente antropizadas pela agricultura, pecuária e urbanização.

O Ribeirão Grande ou da Teteqüera, é um afluente da margem esquerda do rio Paraíba do Sul. Nasce no alto da Serra da Mantiqueira, município de Pindamonhangaba (SP), em região de campos de altitude. Desce as encostas da serra e no trajeto recebe a contribuição dos córregos do Cedro, Canjarana, Ferraz, Convento e Cachoeirão, até desaguar na margem esquerda do rio Paraíba do Sul.

A microbacia do Ribeirão Grande $\left(22^{\circ} 47^{\prime} 08^{\prime \prime}\right.$ 'S, $45^{\circ} 28^{\prime} 17^{\prime \prime} \mathrm{W}$ ) foi amostrada em oito pontos nos córregos Cachoeirão, Cedro, Canjarana, Ferraz e Ribeirão Grande (BraGa, 2004), incluindo desde campos de altitude até a planície do Vale do Paraíba (tab. I). Foram realizadas quatro coletas nos meses de julho e outubro de 2001 e janeiro e abril de 2002.

Em cada período de coleta, por ponto, mensurações de temperatura da água, condutividade, $\mathrm{pH}$ e oxigênio dissolvido foram realizadas, utilizando-se um aparelho Horiba U-10 e as coordenadas e altitude de cada ponto foram obtidas por um receptor GPS.

Foram praticadas três artes de pesca, conforme as condições locais de cada ponto. Foi utilizada uma unidade de pesca elétrica acoplada a uma fonte geradora de eletricidade, que produzia uma corrente elétrica direta de 300 a $500 \mathrm{~V}$ e $8,7 \mathrm{~A}$, percorrendo-se um trecho de 50 metros em cada córrego, com uma repetição; a pesca elétrica não foi realizada no ponto 1 devido à baixa condutividade, nem no ponto 8 , devido à elevada profundidade local. Também foram utilizadas cinco redes de emalhar $(5 \mathrm{~m}$ cada, com malhas 1,$5 ; 2,0 ; 2,5 ; 3,0$ e 3,5 cm entre-nós adjacentes) e três tipos de covos de plástico maleável sendo dois pequenos $(50 \times 8 \mathrm{~cm}$ - comprimento $\times$ abertura), dois médios (64 x $10 \mathrm{~cm}$ - comprimento x abertura) e um grande ( $69 \times 12 \mathrm{~cm}$ - comprimento x abertura). Covos e redes foram armados à tarde e retirados na manhã seguinte. Os peixes coletados eram imediatamente fixados em formol a $10 \%$ e estocados. No laboratório os peixes eram separados por local de coleta, identificados e quantificados.

A diversidade beta de espécies, por local, foi estimada usando-se o coeficiente de comunidade de Jaccard (CC), para análise de presença-ausência de espécies e o índice de Morisita-Horn (Imh), para análise de similaridade da abundância relativa das espécies entre amostras (MAGurRan, 1991; LOHR \& FAusch, 1997). O uso do índice de Morisita-Horn independe do tamanho das amostras e da diversidade das espécies (WoldA, 1981). Valores estimados para o índice de Morisita-Horn menores que 0,50 indicam baixa similaridade na abundância relativa entre espécies e aqueles superiores a 0,75 indicam alta similaridade (MATTHEWs, 1986). Valores estimados para o índice de Jaccard menores que 0,60 indicam substancial diferença na presença/ausência de espécies (RAHEL, 1990).

Tabela I. Descrição dos pontos de coleta na microbacia do Ribeirão Grande, Serra da Mantiqueira Oriental, São Paulo, Brasil.

\begin{tabular}{|c|c|c|c|c|c|}
\hline Ponto & Córrego & Ordem & Coordenadas & Altitude (m) & Ambiente \\
\hline 1 & Cachoeirão & 1 & $22^{\circ} 45,23^{\prime} \mathrm{S} 45^{\circ} 45,03^{\prime} \mathrm{W}$ & 1793 & Campos de altitude \\
\hline 2 & Cedro & 2 & $22^{\circ} 45,03^{\prime} \mathrm{S} 45^{\circ} 27,96^{\prime} \mathrm{W}$ & 900 & Mata pluvial tropical \\
\hline 3 & Cedro & 2 & $22^{\circ} 46,04^{\prime} \mathrm{S} 45^{\circ} 27,83, \mathrm{~W}$ & 671 & Mata pluvial tropical,campos abertos \\
\hline 4 & Canjarana & 3 & $22^{\circ} 46,11^{\prime} \mathrm{S} 45^{\circ} 27,86^{\prime} \mathrm{W}$ & 652 & Campos abertos, pediplano \\
\hline 5 & Ferraz & 2 & $22^{\circ} 47,08^{\prime} \mathrm{S} 45^{\circ} 28,17^{\prime} \mathrm{W}$ & 630 & Campos abertos, pediplano \\
\hline 6 & Ribeirão Grande & 4 & $22^{\circ} 48,41^{\prime} \mathrm{S} 45^{\circ} 26,93, \mathrm{~W}$ & 611 & Campos abertos, pediplano \\
\hline 7 & Ribeirão Grande & 4 & $22^{\circ} 48,99^{\prime} \mathrm{S} 45^{\circ} 25,37^{\prime} \mathrm{W}$ & 577 & Campos abertos, planície do Vale do Paraíba \\
\hline 8 & Ribeirão Grande & 4 & $22^{\circ} 50,89^{\prime} \mathrm{S} 45^{\circ} 21,25^{\prime} \mathrm{W}$ & 538 & Campos abertos, planície do Vale do Paraíba \\
\hline
\end{tabular}

\section{RESULTADOS}

Foi amostrado um total de 2865 peixes, pertencentes a 37 espécies (tab. II). Exemplares testemunhos das espécies encontram-se depositados no Departamento de Zoologia da Universidade Estadual Paulista, campus de Rio Claro. A pesca elétrica foi responsável por $88,7 \%$ dos peixes capturados. Os dados físico-químicos da água e altitudes, por ponto de coleta, encontram-se na figura 1 .

Os pontos amostrados, do topo da encosta até a planície do vale, apresentaram composições distintas quanto à abundância de indivíduos e de espécies. Houve um aumento no número de espécies do ponto 1 ao ponto 6 , ocorrendo em seguida uma queda em direção à planície do vale (tab. II).

As espécies que ocorreram em maior abundância pertencem ao gênero Characidium, onde se reconhecem duas espécies: Characidium lauroi e Characidium alipioi. Houve uma distribuição distinta dessas espécies, com pequena sobreposição em pontos situados no pediplano (tab. II). A terceira espécie mais abundante foi Trichomycterus itatiayae; esta espécie foi mais abundante nos pontos que se situam na transição da 


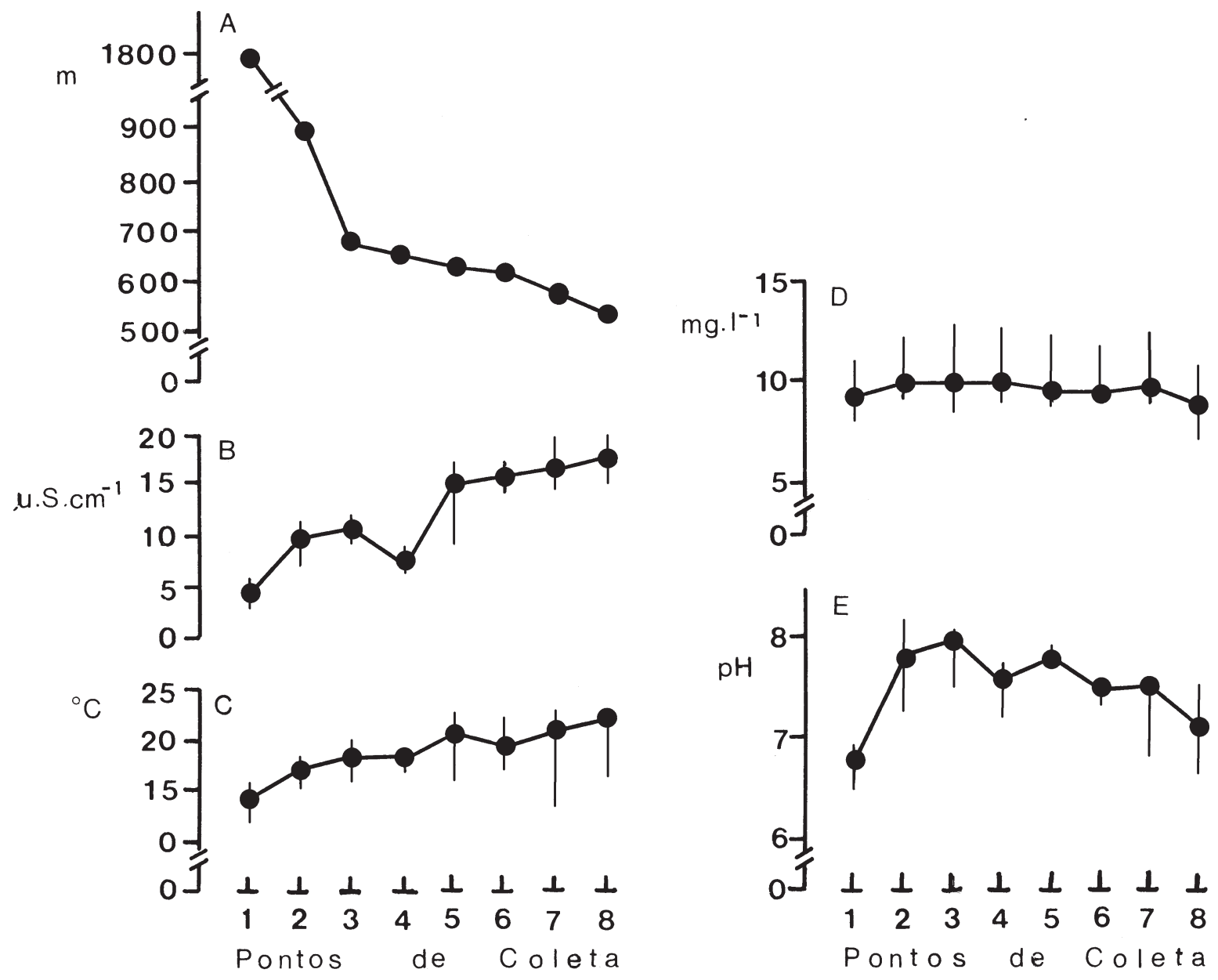

Fig. 1. Representação da altitude (A) e dos valores obtidos para a condutividade (B), temperatura da água (C), oxigênio dissolvido (D) e $\mathrm{pH}(\mathrm{E})$, por ponto de coleta (1 a 8). Os pontos representam os valores medianos e as barras verticais os valores máximos e mínimos obtidos durante as coletas.

encosta com o pediplano. Outras espécies do gênero Trichomycterus foram menos abundantes, distribuindose em córregos do pediplano e estando praticamente ausentes na planície e na encosta (tab. II).

Neoplecostomus micropis e Pareiorhina rudolphi distribuíram-se da encosta ao pediplano. Embora a área de ocorrência dessas espécies tivesse sido a mesma, elas se distribuíram de maneira distinta: Neoplecostomus micropis foi abundante no sopé da encosta e Pareiorhina rudolphi foi abundante na encosta (tab. II).

As demais espécies distribuíram-se do pediplano até a planície do vale (tab. II). Algumas tiveram ocorrência bem definida, como Phalloceros caudimaculatus, que ocorreu só no pediplano. Loricariichthys sp. foi abundante na planície do vale e rara na foz. Imparfinis minutus teve um padrão gradativo no aumento de ocorrência, do pediplano à planície do vale. A distribuição de Harttia carvalhoi, Gymnotus pantherinus e Rhamdia quelen, concentrou-se em pontos do pediplano. Taunayia bifasciata ocorreu na transição da encosta com o pediplano. Outras espécies ocorreram entre o pediplano e a foz, como Astyanax bimaculatus, Pimelodus maculatus, Hypostomus spp. e Gymnotus carapo.

Os índices de diversidade, Jaccard e Morisita-Horn, foram analisados considerando-se pontos em seqüência ou próximos (contíguos) e distantes (não-contíguos). Os pontos 1 e 8 não foram considerados nesta análise por terem sido amostrados com metodologias distintas. $\mathrm{O}$ índice de Jaccard mostrou que somente entre os pontos 4 e 5 houve semelhança entre presença e ausência de espécies, sendo os demais pontos distintos entre si. Quanto à abundância relativa de espécies, analisada pelo índice de Morisita-Horn, os pontos não-contíguos (2 e 7, 3 e 6) apresentaram baixa similaridade, assim como os pontos contíguos 5 e 6 ; a similaridade foi moderada nos pontos contíguos 2 e 3, 4 e 5, e alta nos pontos 3 e 4,6 e 7 (tab. III).

\section{DISCUSSÃO}

Pelas encostas da Serra da Mantiqueira Oriental descem inúmeros riachos que formam microbacias distintas, separadas por cristas de relevo íngreme, que vão desaguar no rio Paraíba do Sul (BRAGA, 2004). A microbacia do Ribeirão Grande está formada por córregos de até quarta ordem, segundo a hierarquia fluvial de Strahler (ChristofoletTI, 1980). Os episódios envolvendo o relevo da Serra da Mantiqueira Oriental, às quais as microbacias têm relações, são recentes e decorrentes de movimentos tectônicos ocorridos no Holoceno, entre 10 
Tabela II. Relação das espécies capturadas por ponto (1 a 8), por ordem de abundância e o total por espécie (TOT) e por ponto. ANO, Anostomidae; CAL, Callichthyidae; CHA, Characidae; CIC, Cichlidae; CRE, Crenuchidae; ERY, Erythrinidae; GYM, Gymnotidae; HEP Heptapteridae; LOR, Loricariidae; PIM, Pimelodidae; POE, Poeciliidae; SYN, Synbranchidae; TRI, Trichomycteridae.

\begin{tabular}{|c|c|c|c|c|c|c|c|c|c|}
\hline Espécie - Família & $\mathrm{P} 1$ & P 2 & P 3 & $\mathrm{P} 4$ & P 5 & P 6 & P 7 & P 8 & TOT \\
\hline Characidium lauroi Travassos, 1949 CRE & & & 127 & 382 & 17 & 6 & & & 532 \\
\hline Characidium alipioi Travassos, 1955 CRE & & & & 3 & 51 & 212 & 254 & & 520 \\
\hline Trichomycterus itatiayae (Miranda-Ribeiro, 1906) TRI & & 43 & 129 & 265 & 44 & 11 & & & 492 \\
\hline Pareiorhina rudolphi (Miranda-Ribeiro, 1911) LOR & & 124 & 64 & 30 & 40 & & & & 258 \\
\hline Neoplecostomus microps (Steindachner, 1877) LOR & & 10 & 22 & 146 & 70 & 1 & & & 249 \\
\hline Astyanax scabripinnis (Jenyns, 1842) CHA & 18 & & 10 & 183 & 18 & 2 & & & 231 \\
\hline Imparfinis minutus (Lütken, 1874) HEP & & & & 8 & 26 & 28 & 38 & & 100 \\
\hline Loricariichthys sp. LOR & & & & & & 6 & 79 & 2 & 87 \\
\hline Harttia carvalhoi Miranda-Ribeiro, 1939 LOR & & & & 15 & 13 & 16 & 2 & & 46 \\
\hline Taunayia bifasciata (Eigenmann \& Norris, 1900) HEP & & & 14 & 29 & & & & & 43 \\
\hline Astyanax bimaculatus (Linnaeus, 1758) CHA & & & & & & 7 & 10 & 25 & 42 \\
\hline Geophagus brasiliensis Kner, 1865 CIC & & & & & 5 & 4 & 23 & 3 & 35 \\
\hline Trichomycterus iheringi (Eigenmann, 1917) TRI & & & & 2 & 23 & 4 & 1 & & 30 \\
\hline Trichomycterus immaculatus (Eigenmann \& Eigenmann, 1889) TRI & & 1 & & 1 & 21 & & & & 23 \\
\hline Pareiorhina sp. 1 LOR & & & 14 & 1 & 2 & 2 & & & 19 \\
\hline Phalloceros caudimaculatus (Hensel, 1868) POE & & & & & 17 & & & & 17 \\
\hline Trichomycterus alternatus (Eigenmann, 1917) TRI & & & & 13 & & & & & 13 \\
\hline Hoplosternum littoralis (Hancock, 1828) CAL & & & & & 1 & & 2 & 9 & 12 \\
\hline Gymnotus pantherinus (Steindachner, 1908) GYM & & & & 1 & 9 & 2 & & & 12 \\
\hline Hypostomus sp. 3 LOR & & & & & & 12 & & & 12 \\
\hline Pimelodus maculatus Lacépède, 1803 PIM & & & & & & 3 & 4 & 3 & 10 \\
\hline Rhamdia quelen (Quoy \& Gaimard, 1824) HEP & & & & 3 & 4 & 2 & & & 9 \\
\hline Astyanax fasciatus (Cuvier, 1819) CHA & & & & & & 5 & & 3 & 8 \\
\hline Trichomycterus sp. TRI & & & & 8 & & & & & 8 \\
\hline Rineloricaria steindachneri (Regan, 1904) LOR & & & & & & 1 & 6 & & 7 \\
\hline Astyanax eigenmanniorum (Cope, 1894) CHA & & & & & & 7 & & & 7 \\
\hline Gymnotus carapo Linnaeus, 1758 GYM & & & & & & 3 & 2 & 2 & 7 \\
\hline Oligosarcus hepsetus (Cuvier, 1829) CHA & & & & & 1 & 1 & & 4 & 6 \\
\hline Hypostomus sp. 2 LOR & & & & & & 6 & & & 6 \\
\hline Hisonotus sp. LOR & & & & & & 5 & & & 5 \\
\hline Pareiorhina sp. 2 LOR & & & & 3 & & 2 & & & 5 \\
\hline Hoplias malabaricus (Bloch, 1794) ERY & & & & & & 2 & 2 & & 4 \\
\hline Hypostomus sp. 1 LOR & & & & & & 3 & & & 3 \\
\hline Synbranchus marmoratus Bloch, 1795 SYN & & & & & & & 3 & & 3 \\
\hline Hypostomus ancistroides (Ihering, 1911) LOR & & & & & & & & 2 & 2 \\
\hline Hoplerythrinus unitaeniatus (Spix \& Agassiz, 1829) ERY & & & & & & & & 1 & 1 \\
\hline Leporinus copelandii Steindachner, 1875 ANO & & & & & & & & 1 & 1 \\
\hline Total & 18 & 178 & 380 & 1093 & 362 & 353 & 426 & 55 & 2865 \\
\hline Número de espécies por ponto & 1 & 4 & 7 & 17 & 17 & 26 & 13 & 11 & \\
\hline
\end{tabular}

mil e 3 mil anos atrás (MODENESI-GAuTtieri et al., 2002). A bacia do Leste, que reúne inúmeros rios que vão desaguar no oceano Atlântico, incluindo o Paraíba do Sul, apresenta um elevado grau de endemismo e tem similaridade faunística com as bacias do Paraná e do São Francisco (BIZERRIL, 1994).

Segundo o conceito de rio contínuo (VAnNote et al., 1980), das cabeceiras à foz forma-se um gradiente de condições físicas que faz com que as populações de organismos aquáticos se ajustem, tendendo a um equilíbrio. As espécies exibem adaptações compatíveis ao hábitat (pequeno porte); morfologia do corpo, como maior desenvolvimento das nadadeiras peitorais (em Crenuchidae), placas ósseas revestindo o corpo (em Loricariidae) e espinhos ósseos operculares (em Trichomycteridae); hábitos alimentares bentófagos (em especial larvas e ninfas de insetos); baixa fecundidade (ovócitos grandes e em pequeno número, redução de gônadas) (BRAGA, 2004). São espécies estrategistas K (PiAnKA, 1974), pois a alocação de recursos alimentares e o espaço físico são limitados nesses ambientes.

$\mathrm{Na}$ distribuição espacial de comunidades de peixes, são importantes os processos que ocorrem ao longo do curso de riachos, na definição da estrutura e funcionamento dessas comunidades (UIEDA \& BARRETTO, 1999), seguindo uma tendência de aumentar a diversidade na direção montante-jusante, em decorrência de uma maior disponibilidade de hábitats (GARutti, 1988). Nos pontos 2, 3 e 4, houve adição de 4, 7 e 12 espécies, elevando o número de espécies da encosta para o pediplano.

O maior número de espécies ocorreu no ponto 6 , que é a transição entre o pediplano e a planície do Vale 
Tabela III. Valores dos índices de Jaccard (CC) e de Morisita-Horn (Imh) estimados para os pontos de coleta contíguos (c) e nãocontíguos (nc).

\begin{tabular}{lll}
\hline PONTOS & \multicolumn{1}{c}{ CC } & \multicolumn{1}{c}{ Imh } \\
\hline $2-3$ (c) & 0,43 (diferentes) & 0,50 (similaridade moderada) \\
$3-4$ (c) & 0,41 (diferentes) & 0,87 (similaridade alta) \\
$4-5$ (c) & 0,62 (semelhantes) & 0,50 (similaridade moderada) \\
$5-6(\mathrm{c})$ & 0,43 (diferentes) & 0,41 (similaridade baixa) \\
$6-7$ (c) & 0,34 (diferentes) & 0,96 (similaridade alta) \\
$2-7$ (nc) & 0,00 (diferentes) & 0,00 (similaridade nula) \\
$3-6$ (nc) & 0,18 (diferentes) & 0,05 (similaridade baixa) \\
\hline
\end{tabular}

do Paraíba. Este ponto, que corresponde ao trecho médio do córrego do Ribeirão Grande, pode ser considerado um ecótono, pois não só a configuração geográfica muda, como também o valor do $\mathrm{pH}$ tende a decrescer e a se elevar os valores da temperatura e da condutividade (fig.1). Peres Neto et al. (1995) encontraram situação semelhante em um rio costeiro no Rio de Janeiro, associando o fato a uma alta heterogeneidade de hábitats.

Riachos de encosta são ambientes frágeis e comportam populações pequenas em número de espécies e abundância de indivíduos, conforme se observa das partes mais altas para as mais baixas. As espécies tendem a ser estrategistas K no conceito de PIANKA (1974), necessitando desenvolver adaptações anatômicas próprias a esses ambientes e fecundidade baixa (BraGA, 2004).

A distribuição de peixes na microbacia do Ribeirão Grande apresenta um padrão de distribuição parapátrico para várias espécies. Esse padrão surge quando espécies de relacionamento muito próximo não sobrepõem suas áreas de distribuição; nesses casos uma espécie é dominante de um lado da linha divisória e a outra é dominante do outro lado (MAYr, 1968; Bull, 1991). A competição interespecífica entre espécies morfologicamente similares pode levar à parapatria, onde também outros fatores bióticos e abióticos contribuem para a manutenção da distribuição parapátrica (TAYLOR \& LIENESCH, 1996). A distribuição da ictiofauna nos riachos de encosta da Serra da Mantiqueira Oriental está associada ao relevo das vertentes, que delimitam e caracterizam as microbacias e às adaptações desenvolvidas pelas espécies de peixes que a habitam.

Características geológicas como a formação do Vale do Paraíba e a elevação das encostas formando a Serra da Mantiqueira no Oligoceno (PETRI \& Fúlfarro, 1983), a remodelagem em períodos recentes das encostas e platô, levando em alguns casos a captura de riachos em cabeceiras (MoDENESI-GAUTTIERI et al., 2002), a existência de fragmentos de Mata Atlântica ao longo das encostas, as elevadas altitudes com ocorrência de baixas temperaturas no inverno, definem particularidades aos riachos que descem as encostas da Serra da Mantiqueira Oriental. O reconhecimento dessas microbacias e suas composições ictiofaunísticas, bem como suas relações, são aspectos importantes que devem ser considerados ao se propor estudos sobre a biologia e ecologia de peixes nesta região.

Agradecimentos. Ao CNPq (proc. no. 304081/85-0) e FAPESP (proc. no. 00/10178-1) pelos auxílios concedidos. Ao Centro de Monitoramento Ambiental da Serra do Itapety e à
Votorantim Celulose e Papel/VCP-Florestal, pelo apoio oferecido durante as atividades de campo na fazenda São Sebastião do Ribeirão Grande. Aos revisores anônimos que analisaram este trabalho.

\section{REFERÊNCIAS BIBLIOGRÁFICAS}

Araúso, F. G. 1996. Composição e estrutura da comunidade de peixes do médio e baixo rio Paraíba do Sul, RJ. Revista Brasileira de Biologia 56:111-126.

Bizerril, C. R. F. S. 1994. Análise taxonômica e biogeográfica da ictiofauna de água-doce do leste brasileiro. Acta Biologica Leopoldinense 16:51-80.

1999. A ictiofauna da bacia do rio Paraíba do Sul. Biodiversidade e padrões biogeográficos. Brazilian Archives of Biology and Technology 42:233-250.

BragA, F. M. S. 2004. Hábitat, distribuição e aspectos adaptativos de peixes da microbacia do Ribeirão Grande, Estado de São Paulo, Brasil. Acta Scientiarum 26:31-36.

Bull, C. M. 1991. Ecology of parapatric distributions. Annual Reviews in Ecology and Systematic 22:19-36.

Christofoletti, A. 1980. Geomorfologia. Rio de Janeiro, Edgar Blücher, 2a . edição. $188 \mathrm{p}$.

DEAn, W. 1996. A ferro e fogo: a história da devastação da Mata Atlântica brasileira. São Paulo, Companhia das Letras. 484 p.

GarutTI, V. 1988. Distribuição longitudinal da ictiofauna de um córrego da região noroeste do Estado de São Paulo, bacia do rio Paraná. Revista Brasileira de Biologia 48:747-758.

Hueck, K. 1972. As florestas da América do Sul. São Paulo, Editora da Universidade de Brasília/Editora Polígono. 466 p.

Lohr, S. C. \& FAusch, K. D. 1997. Multiscale analysis of natural variability in stream fish assemblages of a western great plains watershed. Copeia (4):706-724.

Lundberg, J. G.; Marshal, L. G.; Guerrero, J.; Horton, B.; Malabarba, M. C. S. L. \& Wesseling, F. 1998. The stage for Neotropical fish diversification: a history of tropical South American rivers. In: Malabarba, L. R.; Reis, R. E.; Vari, R. P.; Lucena, Z. M. S. \& Lucena, C. A. S. Phylogeny and classification of Neotropical fishes. Porto Alegre, EDIPUCRS. p.13-48.

Magurran, A. E. 1991. Ecological diversity and its measurement. New York, Chapman \& Hall. 179p.

Matthews, W. J. 1986. Fish faunal structure in an Ozark stream: stability, persistence and a catastrophic flood. Copeia (2):388-397.

Mayr, E. 1968. Especies animales y evolution. Barcelona, Universidad de Chile, Ediciones Ariel. 808p.

Menezes, N. A. 1972. Distribuição e origem da fauna de peixes de água-doce das grandes bacias fluviais do Brasil. In: Comissão Internacional da Bacia Paraná-Uruguai. Poluição e piscicultura. São Paulo, Faculdade de Saúde Pública da USP/ Instituto de Pesca. p.79-108.

1994. A importância da conservação da ictiofauna dos ecossistemas aquáticos brasileiros. In: Comase/Eletrobrás. Seminário sobre fauna aquática e o setor elétrico brasileiro: conservação. Rio de Janeiro, Comase/Eletrobrás. p.7-13.

Modenesi-Gauttieri, M. C.; Hiruma, S. T. \& Riccomini, C. 2002. Morphotectonics of a high plateau on the northwestern flank of the Continental Rift of southeastern Brazil. Geomorphology 43:257-271.

Myers, N.; Mittermeier, R. A.; Mittermeier, C. G.; Fonseca, G. A. B \& Kent, J. 2000. Biodiversity hotspots for conservation priorities. Nature 403:853-858.

Peres Neto, P. R.; Bizerril, C. R. S. F. \& Iglesias, R. 1995. An overview of some aspects of river ecology: a case study on fish assemblages distribution in na eastern brazilian river. In: Esteves, F. A. ed. Oecologia brasiliensis: Estrutura, funcionamento e manejo de ecossistemas brasileiros. Rio de Janeiro, PPGE-UFRJ. v.1, p.317-334.

Petri, S. \& Fúlfarro, V. S. 1983. Geologia do Brasil (Fanerozóico). São Paulo, Edusp. 631p.

Pianka, E. R. 1974. Evolutionary ecology. New York, Harper \& Row. 356p.

Ponçano, W. L.; Carneiro, C. D. R.; Bistrichi, C. A.; Almeida, F. F. M. \& Prandini, F. L. 1981. Mapa geomorfológico do Estado de São Paulo. São Paulo, Instituto de Pesquisas 
Tecnológicas. v.1, p.38-41. (Monografias 5)

RAHEL, F. J. 1990. The hierarchical nature of community persistence: a problem of scale. American Naturalist 136:328-344.

TAYloR, C. M. \& Lienesch, P. W. 1996. Regional parapatry of the congeneric cyprinids Lythrunus snelsoni and L. Umbratilis: species replacement along a complex environmental gradient. Copeia (2):493-497.

Uieda, V. S. \& BARRetto, M. G. 1999. Composição da ictiofauna de quatro trechos de diferentes ordens do rio Capivara, bacia do Tietê, Botucatu, São Paulo. Revista Brasileira de Zoociências 1:55-67.

Vannote, R. L.; Minshall, G. W.; Cummins, K. W.; Sedell, J. R. \& Cushing, C. E. 1980. The river continuum concept. Canadian Journal of Fishery and Aquatic Science 37:130-137.

Wolda, H. 1981. Similarity indices, sample size and diversity. Oecologia 50:296-302. 